\title{
Obeticholic acid for the treatment of non-alcoholic steatohepatitis: interim analysis from a multicentre, randomised, placebo-controlled phase 3 trial
}

\author{
Zobair M Younossi*, Vlad Ratziu*, Rohit Loomba, Mary Rinella, Quentin M Anstee, Zachary Goodman, Pierre Bedossa, Andreas Geier, \\ Susanne Beckebaum, Philip N Newsome, David Sheridan, Muhammad Y Sheikh, James Trotter, Whitfield Knapple, Eric Lawitz, \\ Manal F Abdelmalek, Kris V Kowdley, AldoJ Montano-Loza, Jerome Boursier, Philippe Mathurin, Elisabetta Bugianesi, Giuseppe Mazzella, \\ Antonio Olveira, Helena Cortez-Pinto, Isabel Graupera, David Orr, Lise Lotte Gluud, Jean-Francois Dufour, David Shapiro, Jason Campagna, \\ Luna Zaru, Leigh MacConell, Reshma Shringarpure, Stephen Harrisont, Arun J Sanyalt, on behalf of the REGENERATE Study Investigators
}

\section{Summary}

Background Non-alcoholic steatohepatitis (NASH) is a common type of chronic liver disease that can lead to cirrhosis. Obeticholic acid, a farnesoid X receptor agonist, has been shown to improve the histological features of NASH. Here we report results from a planned interim analysis of an ongoing, phase 3 study of obeticholic acid for NASH.

Methods In this multicentre, randomised, double-blind, placebo-controlled study, adult patients with definite NASH, non-alcoholic fatty liver disease (NAFLD) activity score of at least 4, and fibrosis stages F2-F3, or F1 with at least one accompanying comorbidity, were randomly assigned using an interactive web response system in a 1:1:1 ratio to receive oral placebo, obeticholic acid $10 \mathrm{mg}$, or obeticholic acid $25 \mathrm{mg}$ daily. Patients were excluded if cirrhosis, other chronic liver disease, elevated alcohol consumption, or confounding conditions were present. The primary endpoints for the month-18 interim analysis were fibrosis improvement ( $\geq 1$ stage) with no worsening of NASH, or NASH resolution with no worsening of fibrosis, with the study considered successful if either primary endpoint was met. Primary analyses were done by intention to treat, in patients with fibrosis stage F2-F3 who received at least one dose of treatment and reached, or would have reached, the month 18 visit by the prespecified interim analysis cutoff date. The study also evaluated other histological and biochemical markers of NASH and fibrosis, and safety. This study is ongoing, and registered with ClinicalTrials.gov, NCT02548351, and EudraCT, 20150-025601-6.

Findings Between Dec 9, 2015, and Oct 26, 2018, 1968 patients with stage F1-F3 fibrosis were enrolled and received at least one dose of study treatment; 931 patients with stage F2-F3 fibrosis were included in the primary analysis (311 in the placebo group, 312 in the obeticholic acid $10 \mathrm{mg}$ group, and 308 in the obeticholic acid $25 \mathrm{mg}$ group). The fibrosis improvement endpoint was achieved by 37 (12\%) patients in the placebo group, $55(18 \%)$ in the obeticholic acid $10 \mathrm{mg}$ group $(\mathrm{p}=0.045)$, and $71(23 \%)$ in the obeticholic acid $25 \mathrm{mg}$ group $(\mathrm{p}=0 \cdot 0002)$. The NASH resolution endpoint was not met $(25$ [8\%] patients in the placebo group, 35 [11\%] in the obeticholic acid $10 \mathrm{mg}$ group [p=0 $\cdot 18]$, and 36 [12\%] in the obeticholic acid $25 \mathrm{mg}$ group [ $\mathrm{p}=\mathbf{0} \cdot 13]$ ). In the safety population (1968 patients with fibrosis stages $\mathrm{F} 1-\mathrm{F} 3$ ), the most common adverse event was pruritus (123 [19\%] in the placebo group, 183 [28\%] in the obeticholic acid $10 \mathrm{mg}$ group, and $336[51 \%]$ in the obeticholic acid $25 \mathrm{mg}$ group); incidence was generally mild to moderate in severity. The overall safety profile was similar to that in previous studies, and incidence of serious adverse events was similar across treatment groups $(75[11 \%]$ patients in the placebo group, 72 [11\%] in the obeticholic acid $10 \mathrm{mg}$ group, and $93[14 \%]$ in the obeticholic acid $25 \mathrm{mg}$ group).

Interpretation Obeticholic acid $25 \mathrm{mg}$ significantly improved fibrosis and key components of NASH disease activity among patients with NASH. The results from this planned interim analysis show clinically significant histological improvement that is reasonably likely to predict clinical benefit. This study is ongoing to assess clinical outcomes.

Funding Intercept Pharmaceuticals.

Copyright (c) 2019 Elsevier Ltd. All rights reserved.

\section{Introduction}

Non-alcoholic steatohepatitis (NASH) is an increasingly common cause of chronic liver disease, characterised by hepatocellular injury, inflammation, and progressive fibrosis. Models of disease progression project that the overall burden of end-stage liver disease due to NASH is likely to increase two to three times over the next two decades. ${ }^{1}$ Currently, there are no approved therapies for NASH.

The farnesoid $\mathrm{X}$ receptor is a nuclear receptor that plays a central role in the regulation of bile acids and metabolism. ${ }^{2}$ Recent data indicate that activation of the farnesoid $\mathrm{X}$ receptor can also reduce hepatic fibrosis and inflammation..$^{2-5}$ Previous placebo-controlled clinical
Published Online December 5, 2019 https://doi.org/10.1016/ S0140-6736(19)33041-7

See Online/Comment https://doi.org/10.1016/ S0140-6736(19)32963-0 *Joint first authors tjoint senior authors Betty and Guy Beatty Center for Integrated Research, Inova Health System, Falls Church, VA, USA (Z M Younossi MD, Z Goodman MD); Sorbonne Université, Assistance Publique-Hôpitaux de Paris, Hôpital Pitié-Salpêtrière, Institute for Cardiometabolism and Nutrition, Paris, France (V Ratziu MD); NAFLD Rsearch Center, University of California San Diego, San Diego, CA, USA (R Loomba MD); Feinberg School of Medicine,

Northwestern University, Chicago, IL, USA (M Rinella MD); The Newcastle Liver Research Group, Institute of Cellular Medicine, Faculty of Medical Sciences, Newcastle University, Newcastle upon Tyne, UK (Q M Anstee MD); Newcastle NIHR Biomedical Research Centre, Newcastle upon Tyne Hospitals NHS Foundation Trust, Newcastle Upon Tyne, UK (Q M Anstee); Service d'Anatomie Pathologique, Hôpital Beaujon, Assistance Publique-Hôpitaux de Paris, Paris, France (P Bedossa MD); Department of Hepatology, University of Wuerzburg, Wuerzburg, Germany (A Geier MD); St JosefKrankenhaus Kupferdreh, Essen, Germany (S Beckebaum MD); National Institute for Health Research Biomedical Research Centre, University Hospitals Birmingham NHS Foundation Trust and University of 
Birmingham, Birmingham, UK (PN Newsome MD); Centre for Liver and Gastrointestinal Research, Institute of Immunology and Immunotherapy, University of Birmingham, Birmingham, UK (P N Newsome); Institute of Translational \& Stratified Medicine, University of

Plymouth and University Hospitals Plymouth NHS Trust, Plymouth, UK (D Sheridan MD); Fresno Clinical Research Center, Fresno, CA, USA (M Y Sheikh MD); Baylor Health, Liver Consultants of Texas, Dallas, TX, USA (JTrotter MD); Arkansas Gastroenterology, North Little Rock, AR, USA (W Knapple MD); Texas Liver Institute, University of Texas Health San Antonio, San Antonio, TX, USA (E Lawitz MD); Division of Gastroenterology and Hepatology, Duke University Medical Center, Durham, NC, USA (M F Abdelmalek MD): Swedish Liver Center, Seattle, WA, USA (Prof K V Kowdley MD); Division of Gastroenterology and Liver Unit, University of Alberta, Edmonton, Canada (A) Montano-Loza MD); HIFIH Laboratory, UPRES EA3859, SFR 4208, Angers University, Angers, France (J Boursier MD); Hepato-Gastroenterology Department, Angers University Hospital, Angers, France (J Boursier); Hepatogastroenterology, CHU Lille, Lille, France (P Mathurin MD); Department of Medical Sciences, University of Turin, Turin, Italy (E Bugianesi MD);

Dipartimento di Scienze

Mediche e Chirurgiche, University of Bologna,

Bologna, Italy (G Mazzella MD); Department of

Gastroenterology, Hospital Universitario La Paz, Madrid, Spain (A Olveira MD); Clínica Universitária de

Gastrenterologia, Faculdade de Medicina, Universidade de

Lisboa, Lisbon, Portugal (H Cortez-Pinto MD); Liver Unit, Hospital Clínic de Barcelona, Barcelona, Spain

(I Graupera MD); Institut D'investigacions Biomèdiques August Pi I Sunyer, Barcelona, Spain (I Graupera); Centro de Investigación Biomédica en Red de Enfermedades

Hepáticas y Digestivas, Barcelona, Spain (I Graupera); New Zealand Liver Transplant
Research in context

\section{Evidence before this study}

Non-alcoholic steatohepatitis (NASH) is a chronic progressive liver disease, which can progress to cirrhosis, hepatic decompensation, hepatocellular carcinoma, and liver-related death. Currently, there are no approved therapeutic options for $\mathrm{NASH}$ and treatment is largely limited to lifestyle modifications. We searched PubMed for clinical trials treating NASH published up to Sept 30, 2019, using the terms "nonalcoholic fatty liver disease", "nonalcoholic steatohepatitis", "NAFLD", and "NASH". Early clinical study results for several compounds with various mechanisms of action have shown evidence of improvement in steatohepatitis or fibrosis, but several such studies lacked placebo controls and none of these results have been confirmed in a pivotal phase 3 study setting. The farnesoid $X$ receptor is a nuclear receptor expressed at high levels in the liver. In animal models of liver disease, activation of farnesoid $X$ receptor has been associated with both anti-inflammatory and antifibrotic effects. The placebo-controlled phase 2b FLINT study showed that obeticholic acid, a potent selective farnesoid $X$ receptor agonist, improved key histological features of $\mathrm{NASH}$, including fibrosis. These promising results led to this randomised, placebo-controlled global phase 3 study of obeticholic acid in patients with fibrosis due to NASH (REGENERATE).

\section{Added value of this study}

To our knowledge, REGENERATE is the first positive phase 3 study in patients with NASH. In this interim analysis, a significantly higher proportion of patients treated with obeticholic acid $25 \mathrm{mg}$ had an improvement of fibrosis by at least one stage with no worsening of NASH. Additionally, a post-hoc analysis showed that obeticholic acid treatment

studies have shown that obeticholic acid, a potent and selective farnesoid X receptor agonist, improves glucose disposal after short-term administration ${ }^{6}$ and key histological features of NASH, including fibrosis. ${ }^{7}$ Based on a previous phase 3 study, obeticholic acid was approved for the treatment of primary biliary cholangitis, a progressive autoimmune liver disease, in patients with an inadequate response to, or unable to tolerate, ursodeoxycholic acid. Collectively, this provided a strong rationale for assessing the efficacy and safety of obeticholic acid in patients with NASH and fibrosis in this pivotal phase 3 study.

Liver-related outcomes in patients with $\mathrm{NASH}$ principally occur after the development of cirrhosis; halting progression to cirrhosis is therefore a key treatment goal. Given the length of time to progress to cirrhosis and clinical outcomes, a conditional approval pathway based on demonstration of histological improvement following at least 12 months of treatment is supported by the US Food and Drug Administration (FDA) and the European Medicines Agency, ${ }^{9,10}$

The Randomised Global Phase 3 Study to Evaluate the Impact on NASH with Fibrosis of Obeticholic Acid resulted in NASH resolution with no worsening of fibrosis based on pathologist diagnostic assessment. Obeticholic acid treatment also improved underlying disease activity, as shown by decreased lobular inflammation and hepatocellular ballooning. In addition to improvement in these key histological features, meaningful reduction in laboratory parameters, including robust normalisation of alanine aminotransferase and aspartate aminotransferase, was observed with obeticholic acid treatment. Consistent with previous obeticholic acid clinical studies, pruritus and increased LDL cholesterol were the most commonly reported adverse events. Pruritus incidence was generally mild to moderate in severity and dose dependent. Greater treatment discontinuation was seen in the obeticholic acid $25 \mathrm{mg}$ group, mainly due to protocol requirements. Early increases in LDL cholesterol were observed with obeticholic acid treatment; however, levels approached baseline by month 18 .

\section{Implications of all the available evidence}

Halting progression to cirrhosis, and therefore preventing serious liver-related outcomes, is a key treatment goal in patients with NASH with fibrosis. Advanced liver fibrosis is strongly associated with risk of liver-related adverse outcomes and all-cause mortality, so therapies with proven antifibrotic benefit are highly desirable. Because NASH disease progression occurs over a number of years, assessing clinical outcomes requires long-term evaluation. The positive results of the prespecified REGENERATE month-18 interim analysis are based on surrogate endpoints considered to be reasonably likely to predict clinical benefit, and the study is ongoing through clinical outcomes to confirm long-term benefit.

Treatment (REGENERATE) study is a randomised, controlled, phase 3 study of obeticholic acid in patients with NASH and fibrosis. ${ }^{11}$ Here, we report the results of the prespecified month 18 interim analysis on the safety and efficacy of obeticholic acid in improving fibrosis and underlying disease activity.

\section{Methods}

\section{Study design and participants}

This multicentre, randomised, double-blind, placebocontrolled, phase 3 study is being conducted at 332 centres in 20 countries across the world. Eligible patients were adults (aged $\geq 18$ years) with histological evidence of (per central expert pathologist reading of a liver biopsy obtained $\leq 6$ months from randomisation) definite steatohepatitis; a non-alcoholic fatty liver disease (NAFLD) activity score (NAS) of at least 4, including at least one point for each of steatosis, lobular inflammation, and hepatocellular ballooning; and fibrosis stage per the NASH Clinical Research Network scoring criteria of F2 or F3, or F1 with at least one accompanying comorbidity (obesity [body-mass index $\geq 30 \mathrm{~kg} / \mathrm{m}^{2}$ ], type 2 diabetes, or 
alanine amino transferase [ALT] $>1 \cdot 5 \times$ upper limit of normal [ULN]). Patients were excluded if cirrhosis, other chronic liver disease, elevated alcohol consumption ( $>2$ units/day for women or $>4$ units/day for men for more than 3 consecutive months in the year before screening), or confounding conditions were present; ALT greater than or equal to $10 \times \mathrm{ULN}$; or if they had $\mathrm{HbA}_{1 \mathrm{c}}$ greater than $9.5 \%$ or total bilirubin greater than $1.5 \mathrm{mg} / \mathrm{dL}$.

A planned interim analysis was done after a minimum of 750 randomised patients with fibrosis stages F2 or F3 reached their actual or planned month-18 visit.

Patients were recruited primarily from hepatologists, and from gastroenterologists, academic centres, and community sites. All patients provided written informed consent. This study is being conducted in accordance with the European Union Clinical Trials Directive (2001/20/EC and subsequent amendments), 21 Code of Federal Regulations Part 312, Good Clinical Practice (CPMP/International Councilon Harmonisation/135/95), and with the ethical principles laid down in the Declaration of Helsinki and applicable regulatory requirements. The detailed study design, including inclusion and exclusion criteria, has been previously reported $^{11}$ and a summary of protocol changes can be reviewed on ClinicalTrials.gov.

\section{Randomisation and blinding}

Eligible patients were randomly assigned in a 1:1:1 ratio to receive daily placebo, obeticholic acid $10 \mathrm{mg}$, or obeticholic acid $25 \mathrm{mg}$ orally. Randomisation was based on a predefined randomisation code generated by electronic data capture and done using an interactive web response system; for patients with fibrosis stage F2 or F3, randomisation was stratified by both the presence of type 2 diabetes and the use of thiazolidinediones or vitamin $\mathrm{E}$ at baseline. Placebo and obeticholic acid were supplied as identical tablets in coded containers. All patients, study investigators, and other site research staff were blinded to treatment assignment.

\section{Procedures}

Patients received daily placebo, obeticholic acid $10 \mathrm{mg}$, or obeticholic acid $25 \mathrm{mg}$ orally, in the form of one tablet. Biopsies were obtained at baseline screening and month 18 or end of treatment. Histological assessments followed standardised criteria to ensure consistency, and all biopsies were read centrally. The month-18 (or early termination) biopsy slides were paired with the screening biopsy slides and randomly assigned for reading by one of two central expert liver pathologists (PB and $\mathrm{ZG}$ ), who was masked to both the slide sequence and the patient's treatment. Assessments of liver biochemistry including ALT, aspartate aminotransferase (AST), $\quad \gamma$-glutamyl transferase (GGT), and alkaline phosphatase (ALP) were done at each study visit, which took place every 3 months for the first 18 months.
Additionally, glucose, glycated haemoglobin $\mathrm{A}_{1 \mathrm{c}}$, lipids, and bodyweight were measured every 3 months.

\section{Outcomes}

This study was designed to assess liver histology at month 18 as a surrogate endpoint for clinical outcomes. ${ }^{1}$ The primary endpoints were defined as improvement in fibrosis (reduction of at least one stage) with no worsening of NASH (defined as no increase of hepatocellular ballooning, lobular inflammation, or steatosis), or NASH resolution (defined as the overall histopathologic interpretation of no fatty liver disease or fatty liver disease without steatohepatitis and an NAS of 0 for ballooning and $0-1$ for inflammation) with no worsening of fibrosis. The key secondary endpoint was improvement of fibrosis by at least one stage or resolution of NASH, or both, without worsening of either. Secondary endpoints comprised histological improvement of features of NASH as well as NAS, and liver biochemistry. ${ }^{11}$ A posthoc analysis evaluated NASH resolution on the basis of the pathologist diagnostic assessment of presence or absence of definite steatohepatitis as determined by the overall pattern of injury rather than scoring of individual NAS parameters.

The end-of-study analysis will evaluate the effect of obeticholic acid on clinical outcomes (including progression to cirrhosis and all-cause mortality) and the long-term safety of obeticholic acid, and will be completed once approximately 291 adjudicated clinical outcome events occur. Patients are expected to have a minimum follow-up time of approximately 4 years.

Safety and tolerability of obeticholic acid were assessed by analysis of adverse events, vital signs, electrocardiograms (ECGs), and clinical laboratory assessments (including lipid profile changes); these were all assessed once every 3 months, except for ECGs, which were done on day 1 and at the month-18 visit. Adverse events were graded for severity using Common Terminology Criteria for Adverse Events version 4.03. An independent data monitoring committee reviewed, and continues to review, safety during the study.

\section{Statistical analysis}

For the month-18 interim analysis primary efficacy endpoint of improvement in fibrosis with no worsening of NASH, a sample size of 250 patients per group with an assumed $15 \%$ discontinuation rate was anticipated to provide $98 \%$ power to show a significant treatment difference between the obeticholic acid (10 mg and $25 \mathrm{mg}$ ) and placebo groups based on the CochranMantel-Haenszel test with a two-sided type I error $(\alpha)$ at the 0.01 level, assuming an adjusted response rate of $36.7 \%$ in each of the obeticholic acid groups and $17.6 \%$ in the placebo group. The two-sided type I error allocated to testing both histological endpoints at the month-18 interim analysis is $0 \cdot 02$. Inferential testing was done sequentially in the dose level, adjusting for multiplicity
Unit, Auckland City Hospital, Auckland, New Zealand (D Orr MD); The Gastrounit, Copenhagen University Hospital Hvidovre, Hvidovre, Denmark (L L Gluud MD); University Clinic for Visceral Surgery and Medicine, Inselspital, University of Bern, Bern, Switzerland (J-F Dufour MD); Intercept Pharmaceuticals, San Diego CA, USA (D Shapiro MD, J Campagna MD, L Zaru PhD, L MacConell PhD, R Shringarpure PhD); Pinnacle Clinical Research Center, San Antonio, TX, USA (S Harrison MD); and Department of Internal Medicine, Division of Gastroenterology, Hepatology and Nutrition, Virginia Commonwealth University Richmond, VA, USA (Prof A J Sanyal MD)

Correspondence to: Prof Arun J Sanyal, Department of Internal Medicine, Division of Gastroenterology, Hepatology and Nutrition, Virginia Commonwealth University, Richmond, VA 23298, USA arun.sanyal@vcuhealth.org 


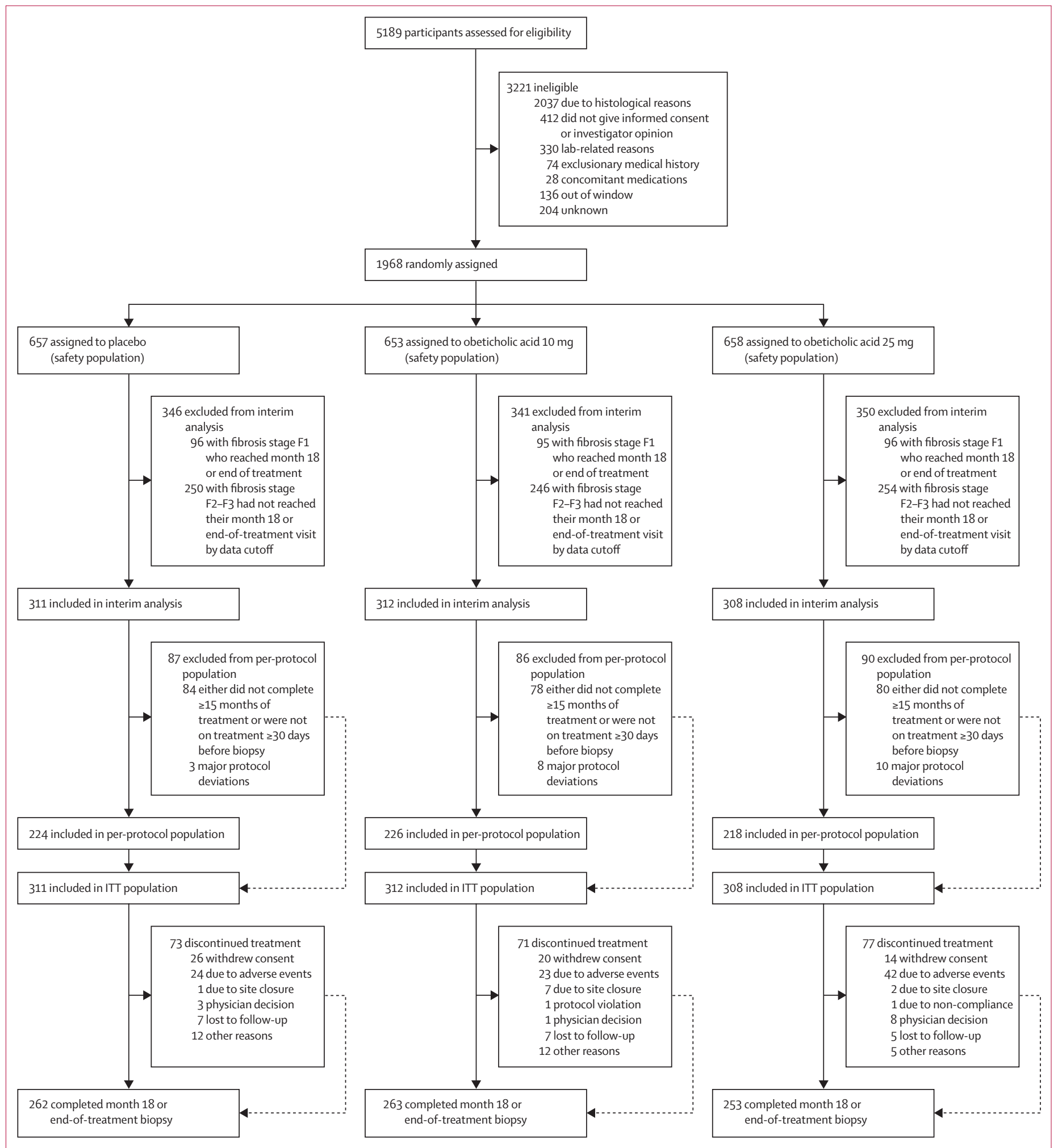

Figure 1: Patient flow diagram

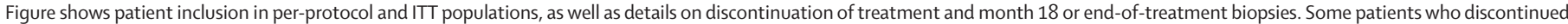
treatment had an end-of-treatment biopsy. ITT=intention to treat. 
using a truncated Hochberg procedure, to test the two primary endpoints within each dose level, starting by comparing the obeticholic acid $25 \mathrm{mg}$ group with placebo for the two primary endpoints, then comparing the obeticholic acid $10 \mathrm{mg}$ group with placebo in the intention-to-treat (ITT) population (appendix pp 2-3). All other testing and the associated $\mathrm{p}$ values reported here are not controlled for type I error and are considered nominal and descriptive. Success of the study was defined as meeting one of the two primary endpoints at the predetermined significance level. For histological endpoints, the comparison between treatment groups was done using the Cochran-MantelHaenszel test stratified by the randomisation strata (type 2 diabetes and use of thiazolidinediones or vitamin $\mathrm{E}$ at baseline [yes vs no]). Continuous endpoints, change from baseline, and percentage change from baseline over time were analysed using a mixed-effect repeated measure model with treatment, baseline, visit, visit by treatment interaction, and stratification factors included in the model. SEs and 95\% CIs were presented by treatment group. The statistical analysis plan, primary endpoints, and requirement for study success were agreed with the FDA before database lock. More information can be found in the appendix (pp 2-3).

All patients (fibrosis stages F1-F3) who received at least one dose of study treatment by the prespecified month-18 interim analysis cutoff date were included in the safety population, which was used for all safety and tolerability analyses. The primary analysis population for efficacy endpoints was the ITT population, comprised of patients with more advanced disease (fibrosis stage F2-F3) who received at least one dose of treatment and reached, or would have reached, the month-18 visit by the prespecified interim analysis cutoff date. Efficacy endpoints were also analysed in the per-protocol population, defined as the ITT population who completed at least 15 months of treatment, had a biopsy at month 18 or at the end of treatment, were on treatment for at least 30 days immediately preceding biopsy, and did not have any major protocol deviations.

This trial was registered with ClinicalTrials.gov, NCT02548351, and EudraCT, 20150-025601-6.

\section{Role of the funding source}

The REGENERATE study was designed by VR, AJS, and ZMY in collaboration with the funder, Intercept Pharmaceuticals, which was involved in data collection, analysis, and interpretation. Operational and protocolspecific aspects were supervised by a steering committee comprising AJS, MR, PB, QMA, RL, SH, VR, ZG, and ZMY (chair). All authors vouch for the fidelity of the study to the protocol, the accuracy and completeness of the data, and approved publication of the manuscript. The first and corresponding authors had full access to the data in the study and had final responsibility for the decision to submit for publication.

\section{Results}

Between Dec 9, 2015, and Oct 26, 2018, 1968 patients were enrolled and randomly assigned to one of the three treatment groups (figure 1). The ITT population included 931 patients randomised to receive placebo ( $\mathrm{n}=311)$, obeticholic acid $10 \mathrm{mg}(\mathrm{n}=312)$, or obeticholic See Online for appendix

\begin{tabular}{|c|c|c|c|}
\hline & $\begin{array}{l}\text { Placebo } \\
(n=311)\end{array}$ & $\begin{array}{l}\text { Obeticholic acid } \\
10 \mathrm{mg}(\mathrm{n}=312)\end{array}$ & $\begin{array}{l}\text { Obeticholic acid } \\
25 \mathrm{mg}(\mathrm{n}=308)\end{array}$ \\
\hline Age, years & $55(12)$ & $55(11)$ & $55(11)$ \\
\hline \multicolumn{4}{|l|}{ Sex } \\
\hline Female & $187(60 \%)$ & $177(57 \%)$ & $175(57 \%)$ \\
\hline Male & $124(40 \%)$ & $135(43 \%)$ & $133(43 \%)$ \\
\hline \multicolumn{4}{|l|}{ Race* $^{*}$} \\
\hline Asian & $10 / 280(4 \%)$ & $17 / 287(6 \%)$ & $20 / 286(7 \%)$ \\
\hline White & $264 / 280(94 \%)$ & 263/287 (92\%) & $249 / 286(87 \%)$ \\
\hline Other & $6 / 280(2 \%)$ & $7 / 287(2 \%)$ & $17 / 286(6 \%)$ \\
\hline \multicolumn{4}{|l|}{ Ethnicity* } \\
\hline Hispanic & $52 / 282(18 \%)$ & $42 / 286(15 \%)$ & 47/282 (17\%) \\
\hline Other & $230 / 282(82 \%)$ & $244 / 286(85 \%)$ & $235 / 282(83 \%)$ \\
\hline \multicolumn{4}{|l|}{ Fibrosis stage } \\
\hline $\mathrm{F} 2$ & $142(46 \%)$ & $130(42 \%)$ & $139(45 \%)$ \\
\hline F3 & $169(54 \%)$ & $182(58 \%)$ & $169(55 \%)$ \\
\hline$N A S \geq 6$ & $215 / 309(70 \%)$ & $211(68 \%)$ & $208(68 \%)$ \\
\hline Type 2 diabetes $†$ & $175(56 \%)$ & $171(55 \%)$ & $171(56 \%)$ \\
\hline Dyslipidaemia & $211(68 \%)$ & $217(70 \%)$ & $205(67 \%)$ \\
\hline Hypertension & $215(69 \%)$ & $215(69 \%)$ & $196(64 \%)$ \\
\hline \multicolumn{4}{|l|}{ Lipids } \\
\hline Total cholesterol, mg/dL & $184 \cdot 5(42 \cdot 7)$ & $185 \cdot 2(53 \cdot 0)$ & $183 \cdot 5(44 \cdot 7)$ \\
\hline HDL cholesterol, mg/dL & $45 \cdot 6(11 \cdot 1)$ & $44 \cdot 9(12 \cdot 1)$ & $44 \cdot 3(11 \cdot 0)$ \\
\hline LDL cholesterol, mg/dL & $114.8(38 \cdot 2)$ & $113.8(38.4)$ & $113 \cdot 3(38 \cdot 8)$ \\
\hline Triglycerides, mg/dL & $178 \cdot 7(154 \cdot 5)$ & $184 \cdot 6(195 \cdot 0)$ & $181 \cdot 7(131 \cdot 6)$ \\
\hline \multicolumn{4}{|l|}{ Metabolic factors } \\
\hline Fasting glucose, mg/dL & $119 \cdot 1(38 \cdot 3)$ & $120 \cdot 8(43 \cdot 6)$ & $119 \cdot 5(40 \cdot 3)$ \\
\hline Bodyweight, kg & $95 \cdot 3(19 \cdot 0)$ & $95 \cdot 2(19 \cdot 1)$ & $95 \cdot 4(19 \cdot 5)$ \\
\hline HOMA-IR & $9 \cdot 6(11 \cdot 8)$ & $9 \cdot 9(16 \cdot 9)$ & $8 \cdot 3(10 \cdot 2)$ \\
\hline $\mathrm{HbA}_{1 \mathrm{c}}$ & $6 \cdot 6 \%(1 \cdot 2)$ & $6 \cdot 5 \%(1 \cdot 2)$ & $6 \cdot 5 \%(1 \cdot 3)$ \\
\hline \multicolumn{4}{|l|}{ Laboratory parameters } \\
\hline $\mathrm{ALT}, \mathrm{U} / \mathrm{L}$ & $79 \cdot 6(56 \cdot 6)$ & $75 \cdot 6(47 \cdot 0)$ & $80 \cdot 2(56 \cdot 4)$ \\
\hline AST, U/L & $58.9(40 \cdot 5)$ & $56 \cdot 6(34 \cdot 0)$ & $57 \cdot 0(34 \cdot 1)$ \\
\hline Platelet count, $\times 10^{9} / \mathrm{L}$ & $241 \cdot 9(67 \cdot 0)$ & $238 \cdot 5(68 \cdot 0)$ & $237 \cdot 2(69 \cdot 0)$ \\
\hline Total bilirubin, mg/dL & $0.64(0.3)$ & $0.65(0.3)$ & $0.69(0.3)$ \\
\hline \multicolumn{4}{|l|}{ Concomitant medication use } \\
\hline Lipid lowering $\ddagger$ & $175(56 \%)$ & $170(54 \%)$ & $160(52 \%)$ \\
\hline Statins & $144(46 \%)$ & $142(46 \%)$ & $127(41 \%)$ \\
\hline Antidiabetic medication & $167(54 \%)$ & $171(55 \%)$ & $159(52 \%)$ \\
\hline Thiazolidinediones $†$ & $5(2 \%)$ & $9(3 \%)$ & $4(1 \%)$ \\
\hline Vitamin E† & $42(14 \%)$ & $34(11 \%)$ & $32(10 \%)$ \\
\hline \multicolumn{4}{|c|}{$\begin{array}{l}\text { Data are } n \text { (\%) or mean (SD). ITT=intention-to-treat. NAS=non-alcoholic fatty liver disease activity score. } \\
\text { HOMA-IR=Homeostatic Model Assessment of Insulin Resistance. ALT=alanine aminotransferase. AST=aspartate } \\
\text { aminotransferase. PCSK9=proprotein convertase subtilisin-kexin type } 9 . \text { *Percentages are calculated on patients for } \\
\text { whom race or ethnicity information was available. †Randomisation was stratified by presence of type } 2 \text { diabetes and } \\
\text { treatment with thiazolidinediones or vitamin E. fLipid-lowering drugs included statins, fibrates, cholesterol-absorbing } \\
\text { resins, PCSK9 inhibitors, and omega-3 fatty acids. }\end{array}$} \\
\hline
\end{tabular}




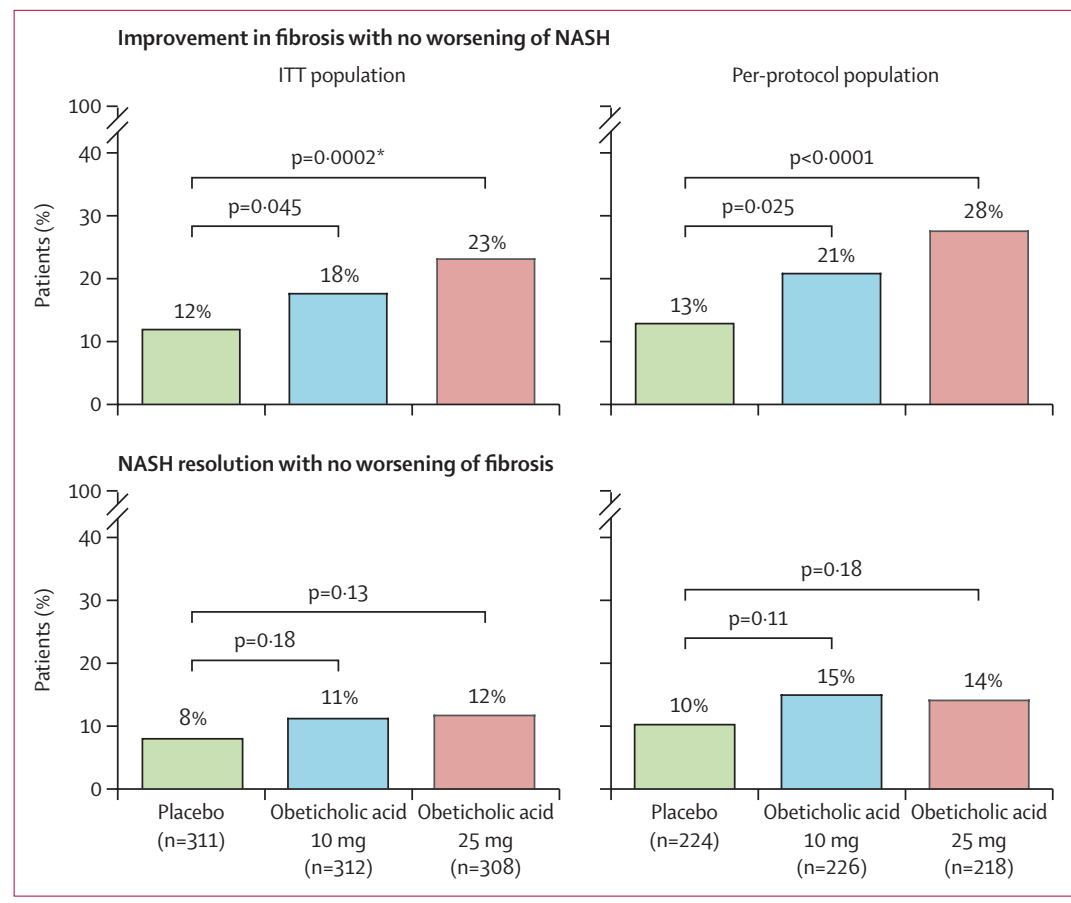

Figure 2: Primary endpoints in the ITT population

The proportion of patients with improvement in fibrosis of at least one stage and no worsening of NASH and the proportion of patients with resolution of NASH and no worsening of fibrosis are shown in the ITT and per-protoco populations. Fibrosis improvement was evaluated per NASH Clinical Research Network criteria; no worsening of NASH defined as no worsening of hepatocellular ballooning, lobular inflammation, or steatosis. NASH resolution defined as overall pathologist assessment of no steatohepatitis, and hepatocellular ballooning $=0$ and lobular inflammation $=0$ or 1 . ITT=intention to treat. $\mathrm{NASH}=$ non-alcoholic steatohepatitis. *Significant in accordance with the statistical analysis plan as agreed with the US Food and Drug Administration.

acid $25 \mathrm{mg}(\mathrm{n}=308)$. At the time of the interim analysis, $73(23 \%)$ patients in the placebo group, $71(23 \%)$ in the obeticholic acid $10 \mathrm{mg}$ group, and 77 (25\%) in the obeticholic acid $25 \mathrm{mg}$ group had discontinued treatment (figure 1); 252 (81\%) patients receiving placebo, 253 (81\%) receiving obeticholic acid $10 \mathrm{mg}$, and 243 (79\%) receiving obeticholic acid $25 \mathrm{mg}$ completed the month 18 biopsy. An additional 3\% of patients in each treatment group (ten patients in each group) completed any post-baseline biopsy (ie, patients who discontinued treatment before month 18 and underwent an end-of-treatment biopsy). The per-protocol population included 668 patients and the safety population included 1968 patients.

In the ITT population, baseline characteristics seemed balanced across treatment groups and reflective of a non-cirrhotic NASH population (table 1). A majority of patients had stage F3 fibrosis (54-58\%) and NAS of at least 6 (68-70\%), indicative of advanced fibrosis and high disease activity. Consistent with NASH epidemiology, more than half of patients had type 2 diabetes (55-56\%), and $52-55 \%$ overall were receiving antidiabetic medication at baseline. Additionally, $41-46 \%$ of patients were receiving statin therapy and a minority were receiving NASH-modifying agents, thiazolidinediones (1-3\%) and vitamin E (10-14\%). A similar pattern of baseline characteristics was observed in the per-protocol population (appendix p 6).

The primary endpoint of fibrosis improvement by at least one stage with no worsening of NASH was met by $37(12 \%)$ patients in the placebo group, $55(18 \%)$ patients in the obeticholic acid $10 \mathrm{mg}$ group ( $\mathrm{p}=0.045 \mathrm{vs}$ placebo), and $71(23 \%)$ patients in the obeticholic acid $25 \mathrm{mg}$ group ( $\mathrm{p}=0.0002 v \mathrm{v}$ placebo) with an obeticholic acid-to-placebo response ratio of $1 \cdot 5(95 \% \mathrm{CI} 1 \cdot 0-2 \cdot 2)$ for the obeticholic $10 \mathrm{mg}$ group and $1.9(1.4-2 \cdot 8)$ for the obeticholic acid $25 \mathrm{mg}$ group (figure 2; table 2). Obeticholic acid $25 \mathrm{mg}$ was significant per the prespecified inferential testing method. Similar results were observed in the perprotocol population (figure 2; table 2). Across subgroups of interest in the ITT population, an improvement of at least one stage in fibrosis with no worsening of NASH was observed in the obeticholic acid $25 \mathrm{mg}$ group (appendix p 7). Several of the subgroup analyses (ie, use of thiazolidinediones or vitamin E, race, and age) were limited by imbalances in sample sizes within a given subgroup to an extent that precluded meaningful comparison (appendix p 7).

In the per-protocol population, which includes patients with at least 15 months of treatment, three times as many patients achieved an improvement in fibrosis of at least one stage compared with progression of fibrosis in the obeticholic acid $25 \mathrm{mg}$ group (81 [38\%] vs 23 [13\%]); in the placebo group, a similar number of patients improved (51 [23\%]) or worsened (46 [21\%]; figure 3). This analysis suggests that on a placebo-adjusted basis, after 18 months of treatment, four to five patients with NASH and fibrosis stage F2-F3 would need to be treated with obeticholic acid $25 \mathrm{mg}$ for one such patient to achieve either improvement ( $\geq 1$ stage) or no worsening of fibrosis.

The primary endpoint of NASH resolution (based on no hepatocellular ballooning and no residual lobular inflammation) with no worsening of fibrosis did not meet statistical significance in the ITT population (25 [8\%] patients in the placebo group vs 35 [11\%] in the obeticholic acid $10 \mathrm{mg}$ group [p=0.18] or 36 [12\%] in the obeticholic acid $25 \mathrm{mg}$ group [p=0.13]), with a response ratio of $1.4(95 \%$ CI $0 \cdot 9-2 \cdot 3)$ for obeticholic acid $10 \mathrm{mg}$ and $1.5(0.9-2.4)$ for obeticholic acid $25 \mathrm{mg}$ (figure 2 table 2). Similar results were observed in the per-protocol population (figure 2; table 2). Despite not meeting the NASH resolution endpoint, a dose-dependent response was observed in the ITT population, with more patients in the obeticholic acid $25 \mathrm{mg}$ group showing at least a 1-point improvement in scores in key histological features of NASH compared with the placebo group (136 [44\%] patients vs 111 [36\%] for lobular inflammation [p=0.032] and 108 [35\%] vs 72 [23\%] for hepatocellular ballooning $[\mathrm{p}=0 \cdot 0011]$; table 2 ; appendix $\mathrm{p} 8$ ).

In a post-hoc analysis, NASH resolution was evaluated by assessing a change from presence of definite steatohepatitis at baseline to absence of definite steatohepatitis 


\begin{tabular}{|c|c|c|c|c|c|c|c|c|c|c|}
\hline & \multicolumn{5}{|c|}{ ITT population $(\mathrm{N}=931)$} & \multicolumn{5}{|c|}{ Per-protocol population $(\mathrm{N}=668)$} \\
\hline & \multirow[t]{2}{*}{$\begin{array}{l}\text { Placebo } \\
(n=311)\end{array}$} & \multicolumn{2}{|c|}{$\begin{array}{l}\text { Obeticholic acid } 10 \mathrm{mg} \\
(\mathrm{n}=312)\end{array}$} & \multicolumn{2}{|c|}{$\begin{array}{l}\text { Obeticholic acid } 25 \mathrm{mg} \\
(\mathrm{n}=308)\end{array}$} & \multirow[t]{2}{*}{$\begin{array}{l}\text { Placebo } \\
(\mathrm{n}=224)\end{array}$} & \multicolumn{2}{|c|}{$\begin{array}{l}\text { Obeticholic acid } 10 \mathrm{mg} \\
(n=226)\end{array}$} & \multicolumn{2}{|c|}{$\begin{array}{l}\text { Obeticholic acid } 25 \mathrm{mg} \\
(\mathrm{n}=218)\end{array}$} \\
\hline & & Patients & $\begin{array}{l}\mathrm{RR}(95 \% \mathrm{Cl}) \\
\text { p value }\end{array}$ & Patients & $\begin{array}{l}\mathrm{RR}(95 \% \mathrm{Cl}) \\
\text { p value }\end{array}$ & & Patients & $\begin{array}{l}\mathrm{RR}(95 \% \mathrm{Cl}) \\
\text { p value }\end{array}$ & Patients & $\begin{array}{l}\text { RR }(95 \% \mathrm{Cl}) \\
\text { p value }\end{array}$ \\
\hline \multicolumn{11}{|l|}{ Primary endpoints } \\
\hline $\begin{array}{l}\text { Improvement of fibrosis with no } \\
\text { worsening of NASH }\end{array}$ & $37(12 \%)$ & $55(18 \%)$ & $\begin{array}{l}1 \cdot 5(1 \cdot 0-2 \cdot 2) \\
\mathrm{p}=0 \cdot 045\end{array}$ & $71(23 \%)$ & $\begin{array}{l}1 \cdot 9(1 \cdot 4-2 \cdot 8) \\
p=0 \cdot 0002\end{array}$ & $29(13 \%)$ & $47(21 \%)$ & $\begin{array}{l}1 \cdot 6(1 \cdot 1-2 \cdot 5) \\
p=0.025\end{array}$ & $60(28 \%)$ & $\begin{array}{l}2 \cdot 2(1 \cdot 4-3 \cdot 2) \\
p<0 \cdot 0001\end{array}$ \\
\hline $\begin{array}{l}\text { Resolution of NASH with no worsening } \\
\text { of fibrosis }\end{array}$ & $25(8 \%)$ & $35(11 \%)$ & $\begin{array}{l}1 \cdot 4(0 \cdot 9-2 \cdot 3) \\
\mathrm{p}=0.18\end{array}$ & $36(12 \%)$ & $\begin{array}{l}1 \cdot 5(0 \cdot 9-2 \cdot 4) ; \\
p=0.13\end{array}$ & $23(10 \%)$ & $34(15 \%)$ & $\begin{array}{l}1 \cdot 5(0 \cdot 9-2 \cdot 4) \\
p=0 \cdot 11\end{array}$ & $31(14 \%)$ & $\begin{array}{l}1 \cdot 4(0 \cdot 9-2 \cdot 3) \\
\mathrm{p}=0.18\end{array}$ \\
\hline \multicolumn{11}{|l|}{ Secondary endpoints* } \\
\hline $\begin{array}{l}\text { Improvement of fibrosis by } \geq 1 \text { stage or } \\
\text { resolution of NASH without worsening } \\
\text { of either }\end{array}$ & $49(16 \%)$ & $67(21 \%)$ & $\begin{array}{l}1 \cdot 4(1 \cdot 0-1 \cdot 9) \\
\mathrm{p}=0 \cdot 068\end{array}$ & $84(27 \%)$ & $\begin{array}{l}1 \cdot 7(1 \cdot 3-2 \cdot 4) \\
p=0 \cdot 0005\end{array}$ & $41(18 \%)$ & $59(26 \%)$ & $\begin{array}{l}1 \cdot 4(1 \cdot 0-2 \cdot 1) \\
p=0 \cdot 041\end{array}$ & $71(33 \%)$ & $\begin{array}{l}1.8(1.3-2 \cdot 5) ; \\
p=0.0004\end{array}$ \\
\hline $\begin{array}{l}\text { No worsening of fibrosis and no } \\
\text { worsening of NASH }\end{array}$ & $117(38 \%)$ & $127(41 \%)$ & $\begin{array}{l}1 \cdot 1(0 \cdot 9-1 \cdot 3) \\
\mathrm{p}=0 \cdot 43\end{array}$ & $147(48 \%)$ & $\begin{array}{l}1 \cdot 3(1 \cdot 1-1 \cdot 5) \\
p=0 \cdot 011\end{array}$ & $100(45 \%)$ & $109(48 \%)$ & $\begin{array}{l}1 \cdot 1(0 \cdot 9-1 \cdot 3) \\
p=0 \cdot 43\end{array}$ & $125(57 \%)$ & $\begin{array}{l}1.3(1.1-1.6) \\
p=0.0062\end{array}$ \\
\hline $\begin{array}{l}\text { Improvement of NAS by } \geq 2 \text { with no } \\
\text { worsening of fibrosis }\end{array}$ & $76(24 \%)$ & $94(30 \%)$ & $\begin{array}{l}1 \cdot 2(1 \cdot 0-1 \cdot 6) \\
\mathrm{p}=0 \cdot 11\end{array}$ & $112(36 \%)$ & $\begin{array}{l}1 \cdot 5(1 \cdot 2-1 \cdot 9) \\
p=0.0012\end{array}$ & $69(31 \%)$ & $82(36 \%)$ & $\begin{array}{l}1 \cdot 2(0 \cdot 9-1 \cdot 5) \\
p=0 \cdot 19\end{array}$ & $96(44 \%)$ & $\begin{array}{l}1.4(1.1-1.8) \\
\mathrm{p}=0.0035\end{array}$ \\
\hline $\begin{array}{l}\text { Improvement of fibrosis and resolution } \\
\text { of NASH as a composite endpoint } \dagger\end{array}$ & $13(4 \%)$ & $23(7 \%)$ & $\begin{array}{l}1.8(0.9-3 \cdot 4) \\
p=0.090\end{array}$ & $23(7 \%)$ & $\begin{array}{l}1 \cdot 8(0 \cdot 9-3 \cdot 4) \\
p=0.080\end{array}$ & $11(5 \%)$ & $22(10 \%)$ & $\begin{array}{l}2 \cdot 0(1 \cdot 0-4 \cdot 1) \\
p=0 \cdot 045\end{array}$ & $20(9 \%)$ & $\begin{array}{l}1.9(1.0-3 \cdot 9) \\
p=0.064\end{array}$ \\
\hline Improvement in fibrosis by $\geq 2$ stages & $15(5 \%)$ & $19(6 \%)$ & $\begin{array}{l}1 \cdot 3(0 \cdot 7-2 \cdot 4) \\
p=0 \cdot 49\end{array}$ & $30(10 \%)$ & $\begin{array}{l}2 \cdot 0(1 \cdot 1-3 \cdot 7) \\
p=0.018\end{array}$ & $10(4 \%)$ & $16(7 \%)$ & $\begin{array}{l}1 \cdot 6(0 \cdot 8-3 \cdot 5) \\
p=0 \cdot 22\end{array}$ & $29(13 \%)$ & $\begin{array}{l}3.1(1.5-6 \cdot 1) ; \\
p=0.0008\end{array}$ \\
\hline Resolution of fibrosis & $4(1 \%)$ & $8(3 \%)$ & $\begin{array}{l}2 \cdot 0(0.6-6 \cdot 4) \\
p=0 \cdot 25\end{array}$ & $10(3 \%)$ & $\begin{array}{l}2 \cdot 5(0 \cdot 8-7 \cdot 9) \\
p=0 \cdot 10\end{array}$ & $4(2 \%)$ & $8(4 \%)$ & $\begin{array}{l}2 \cdot 1(0.6-6 \cdot 7) \\
p=0.21\end{array}$ & $9(4 \%)$ & $\begin{array}{l}2 \cdot 4(0 \cdot 7-7 \cdot 6) \\
p=0 \cdot 14\end{array}$ \\
\hline$\geq 1$-point improvement in steatosis & $118(38 \%)$ & $127(41 \%)$ & $\begin{array}{l}1 \cdot 1(0 \cdot 9-1 \cdot 3) \\
\mathrm{p}=0 \cdot 49\end{array}$ & $127(41 \%)$ & $\begin{array}{l}1 \cdot 1(0 \cdot 9-1 \cdot 3) \\
p=0 \cdot 40\end{array}$ & $97(43 \%)$ & $108(48 \%)$ & $\begin{array}{l}1 \cdot 1(0 \cdot 9-1 \cdot 4) \\
p=0 \cdot 33\end{array}$ & $113(52 \%)$ & $\begin{array}{l}1 \cdot 2(1.0-1 \cdot 5) ; \\
p=0.072\end{array}$ \\
\hline $\begin{array}{l}\text { 21-point improvement in lobular } \\
\text { inflammation }\end{array}$ & $111(36 \%)$ & $123(39 \%)$ & $\begin{array}{l}1 \cdot 1(0 \cdot 9-1 \cdot 4) \\
p=0 \cdot 34\end{array}$ & $136(44 \%)$ & $\begin{array}{l}1 \cdot 2(1 \cdot 0-1 \cdot 5) \\
p=0 \cdot 032\end{array}$ & $94(42 \%)$ & $104(46 \%)$ & $\begin{array}{l}1 \cdot 1(0 \cdot 9-1 \cdot 4) \\
p=0 \cdot 38\end{array}$ & 114 (52\%) & $\begin{array}{l}1 \cdot 3(1.0-1.5) ; \\
p=0.031\end{array}$ \\
\hline $\begin{array}{l}\geq 1 \text {-point improvement in hepatocellular } \\
\text { ballooning }\end{array}$ & $72(23 \%)$ & $85(27 \%)$ & $\begin{array}{l}1 \cdot 2(0 \cdot 9-1 \cdot 5) \\
\mathrm{p}=0 \cdot 24\end{array}$ & $108(35 \%)$ & $\begin{array}{l}1 \cdot 5(1 \cdot 2-2 \cdot 0) \\
p=0 \cdot 0011\end{array}$ & $64(29 \%)$ & $77(34 \%)$ & $\begin{array}{l}1 \cdot 2(0 \cdot 9-1 \cdot 6) \\
\mathrm{p}=0 \cdot 19\end{array}$ & $95(44 \%)$ & $\begin{array}{l}1.5(1.2-2.0) ; \\
p=0.0008\end{array}$ \\
\hline $\begin{array}{l}\text { Pathologist assessment of NASH } \\
\text { resolution with no worsening of fibrosisł }\end{array}$ & $38(12 \%)$ & $51(16 \%)$ & $\begin{array}{l}1 \cdot 3(0 \cdot 9-2 \cdot 0) \\
p=0 \cdot 14\end{array}$ & $71(23 \%)$ & $\begin{array}{l}1 \cdot 9(1 \cdot 3-2 \cdot 7) \\
p=0.0004\end{array}$ & $35(16 \%)$ & $48(21 \%)$ & $\begin{array}{l}1 \cdot 4(0 \cdot 9-2 \cdot 0) \\
p=0 \cdot 11\end{array}$ & $63(29 \%)$ & $\begin{array}{l}1.9(1.3-2 \cdot 7) \\
p=0.0005\end{array}$ \\
\hline $\begin{array}{l}\text { ITT=intention-to-treat. RR=response ratio. } \\
\text { Cochran-Mantel-Haenszel test, stratified by } \\
\text { composite endpoint where both of the prin } \\
\text { definite steatohepatitis at baseline to abser }\end{array}$ & 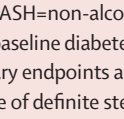 & 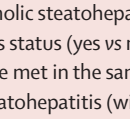 & w & (1) & $\begin{array}{l}\text { H resoluti } \\
\text { h } 18 .\end{array}$ & led by & ologis & all assessn & hange & $\begin{array}{l}\text { sing the } \\
\text { is defined as a } \\
\text { resence of }\end{array}$ \\
\hline
\end{tabular}

(without worsening of fibrosis) at month 18. This pathologist diagnostic assessment of NASH, based on the overall pattern of liver injury, showed that in the ITT population approximately twice as many patients in the obeticholic acid $25 \mathrm{mg}$ group achieved NASH resolution compared with the placebo group (71 [23\%] vs 38 [12\%], $\mathrm{p}=0.0004$; appendix $\mathrm{p}$ 9). A similar dose-dependent response was observed in the per-protocol population (63 [29\%] vs 35 [16\%], p=0 -0005; appendix p 9).

The key secondary endpoint of improvement of fibrosis of at least one stage or resolution of $\mathrm{NASH}$, without worsening of either, was observed in $49(16 \%)$ patients in the placebo group, 67 (21\%) in the obeticholic acid $10 \mathrm{mg}$ group, and 84 (27\%) in the obeticholic acid $25 \mathrm{mg}$ patients in the ITT population (table 2; appendix p 10). A significantly higher proportion of patients receiving obeticholic acid 25 mg compared with placebo showed improvement in NAS by at least two points with no worsening of fibrosis, had no disease progression as assessed by no worsening of fibrosis and no worsening of $\mathrm{NASH}$, and had improvement in fibrosis of at least two stages (table 2). Additional secondary NASH and fibrosis endpoints are provided in table 2.

Favourable changes in key liver enzymes were observed in patients treated with obeticholic acid. Early dosedependent decreases in ALT and AST were observed by month 3 and continued through month 18 (mean change from baseline at month 18 in ALT: -15.6 U/L [SE 3.3] for the placebo group, $-23.8 \mathrm{U} / \mathrm{L}$ [2.6] for the obeticholic acid $10 \mathrm{mg}$ group, and $-36 \cdot 0 \mathrm{U} / \mathrm{L}$ [3.6] for the obeticholic acid $25 \mathrm{mg}$ group; mean change in AST of $-9.8 \mathrm{U} / \mathrm{L}$ [2.4] for the placebo group, $-14 \cdot 1 \mathrm{U} / \mathrm{L}[2 \cdot 1]$ for the obeticholic acid $10 \mathrm{mg}$ group, and $-20 \cdot 4 \mathrm{U} / \mathrm{L}$ [2.3] for the obeticholic acid $25 \mathrm{mg}$ group; figure 4). These changes correspond to a decrease in ALT of $6 \%$ for placebo, $26 \%$ for obeticholic acid $10 \mathrm{mg}$, and $33 \%$ for obeticholic acid $25 \mathrm{mg}$ and in AST of $4 \%, 19 \%$, and $24 \%$ (figure 4). A post-hoc analysis showed that a higher proportion of patients receiving obeticholic acid with elevated ALT and AST at baseline achieved levels below the ULN at month 18 compared 


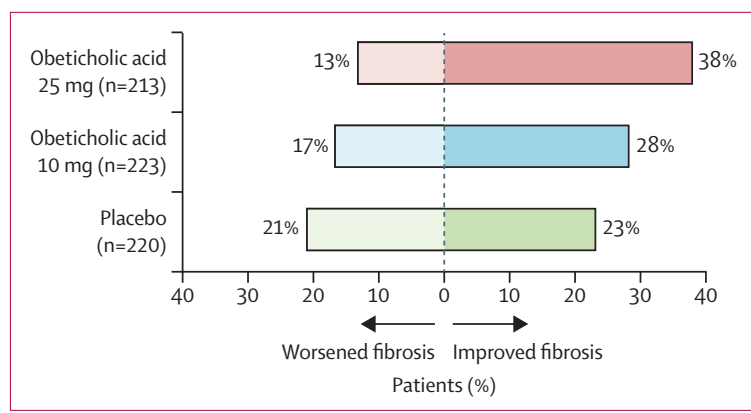

Figure 3: Regression or progression of fibrosis by at least one stage in the per-protocol population

The proportion of patients with improved or worsened fibrosis by at least one stage is shown for the 656 patients in the per-protocol population with available fibrosis stage data at month 18 or end of treatment.
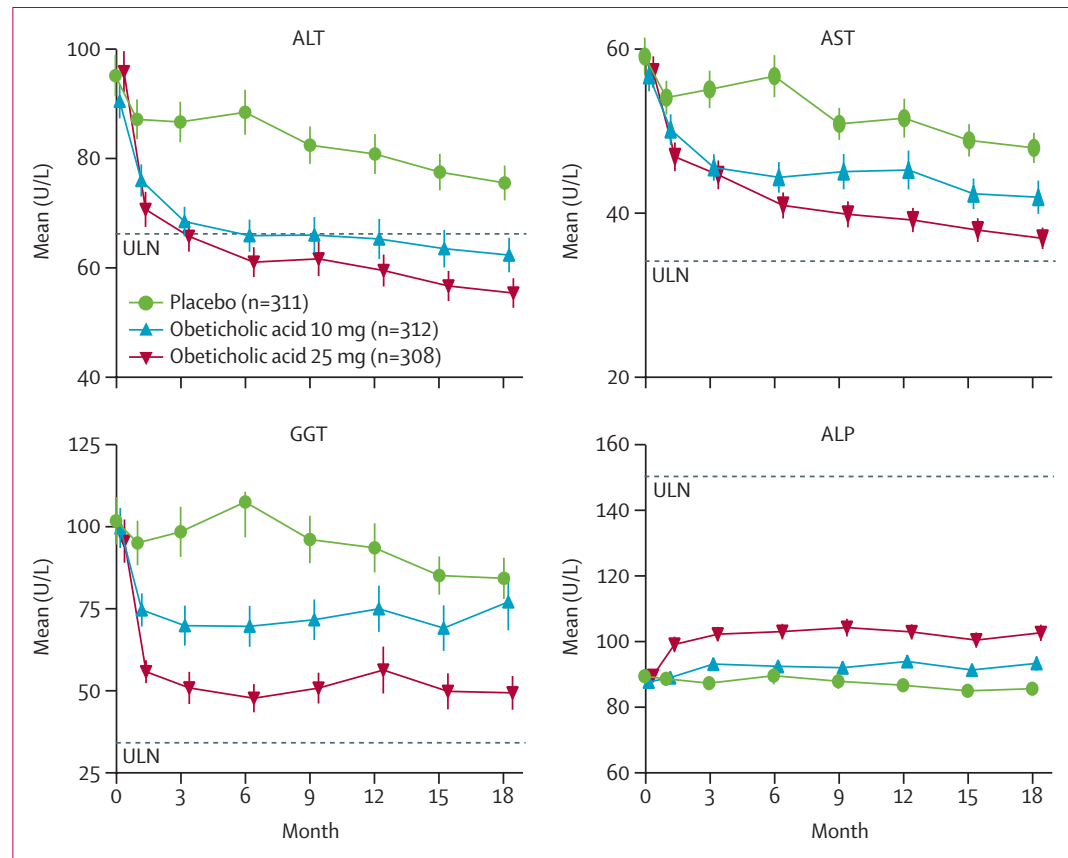

Figure 4: Changes in liver biochemistry over time in the ITT population

Mean values of change from baseline up to month 18 are shown for patients from each treatment group in the ITT population, with vertical bars indicating SEs. ALP=alkaline phosphatase; $A L T=$ alanine aminotransferase.

AST=aspartate aminotransferase. $G G T=\gamma$-glutamyl transferase. ITT=intention to treat. $U L N=$ upper limit of normal.

with placebo (appendix p 11). GGT levels declined rapidly and were generally stable after month 3 (change at month 18: $1 \%$ increase for the placebo group, $24 \%$ decrease for the obeticholic acid $10 \mathrm{mg}$ group, and $38 \%$ decrease for the obeticholic acid $25 \mathrm{mg}$ group; figure 4). Increases in ALP were observed with obeticholic acid treatment, but levels remained below the ULN throughout the study period (change at month 18: $1 \%$ decrease for the placebo group, $9 \%$ increase for the obeticholic acid $10 \mathrm{mg}$ group, and $20 \%$ increase for the obeticholic acid $25 \mathrm{mg}$ group; figure 4).

Additionally, treatment with obeticholic acid resulted in a dose-dependent decrease in bodyweight at month 18 (mean change from baseline $-0.7 \mathrm{~kg}$ [SE 0.4] for the placebo group, $-1.8 \mathrm{~kg}[0.4]$ for the obeticholic acid $10 \mathrm{mg}$ group, and $-2 \cdot 2 \mathrm{~kg}[0 \cdot 3]$ for the obeticholic acid $25 \mathrm{mg}$ group).

1968 patients were included in the safety population, comprised of fibrosis stage F1 (290 [15\%] patients), stage F2 (698 [35\%]), and stage F3 (980 [50\%]; figure 1). The duration of exposure was generally similar across treatment groups. Overall, treatment-emergent adverse events occurred in 548 (83\%) patients in the placebo group, 579 (89\%) in the obeticholic acid $10 \mathrm{mg}$ group, and 601 (91\%) in the obeticholic acid $25 \mathrm{mg}$ group (table 3). Most treatment-related adverse events were of mild or moderate severity (table 3). The frequency of serious adverse events was similar across treatment groups (11-14\%) and no single serious adverse event occurred in more than $1 \%$ of patients in any treatment group (table 3 ). The most frequent adverse event was pruritus (table 3). The incidence of pruritus was highest during the first 3 months of treatment with obeticholic acid, and generally mild to moderate in severity. Treatment discontinuation due to pruritus occurred in five $(<1 \%)$ patients in the placebo group, five $(<1 \%)$ in the obeticholic acid $10 \mathrm{mg}$ group, and 57 (9\%) in the obeticholic acid $25 \mathrm{mg}$ group. Of the 57 patients in the obeticholic acid $25 \mathrm{mg}$ group who discontinued due to pruritus, 36 discontinuations were protocol mandated based on the investigator-assessed grade of the event.

In patients receiving obeticholic acid, LDL cholesterol increased by month 1 (mean change from baseline $-3.0 \mathrm{mg} / \mathrm{dL}$ [SE 0.9] in the placebo group, $17.8 \mathrm{mg} / \mathrm{dL}$ [1.0] in the obeticholic acid $10 \mathrm{mg}$ group, and $23.8 \mathrm{mg} / \mathrm{dL}[1 \cdot 1]$ in the obeticholic acid $25 \mathrm{mg}$ group) and decreased thereafter, approaching baseline by month 18 (mean change from baseline $-7.1 \mathrm{mg} / \mathrm{dL}$ [1.7] for the placebo group, $1.4 \mathrm{mg} / \mathrm{dL}$ [2.0] for the obeticholic acid $10 \mathrm{mg}$ group, and $2 \cdot 7 \mathrm{mg} / \mathrm{dL}$ [2.1] for the obeticholic acid $25 \mathrm{mg}$ group; appendix p 12). 380 patients started statin therapy during the study (66 in the placebo group, 155 in the obeticholic acid $10 \mathrm{mg}$ group, and 159 in the obeticholic acid $25 \mathrm{mg}$ group). Among obeticholic acidtreated patients who initiated statins, the initial LDL cholesterol increases reversed to below baseline levels as of month 6 and were sustained through month 18 (appendix p 13). There was no clear pattern of fibrosis improvement by statin use. Levels of HDL cholesterol showed dose-dependent decreases by month 1 (mean change from baseline $-0.7 \mathrm{mg} / \mathrm{dL}[0 \cdot 2]$ in the placebo group, $-1.8 \mathrm{mg} / \mathrm{dL}[0 \cdot 2]$ in the obeticholic acid $10 \mathrm{mg}$ group, and $-4.6 \mathrm{mg} / \mathrm{dL}[0.3]$ in the obeticholic acid $25 \mathrm{mg}$ group) and were sustained through month 18 mean HDL cholesterol remained within the normal limit (>40 mg/dL) at all timepoints (appendix $\mathrm{p} \mathrm{12}$ ). Changes in total cholesterol over time were similar to those for LDL cholesterol (appendix p 12). A dosedependent decrease in triglycerides was observed by month 1 in the obeticholic acid groups, with levels continuing to decline with a maximum mean change 
from baseline of $-37.4 \mathrm{mg} / \mathrm{dL}$ in the obeticholic acid $25 \mathrm{mg}$ group at month 18 (appendix p 12).

The incidence of cardiovascular adverse events and serious adverse events was similar across treatment groups (adverse events: 30 [5\%] in the placebo group, 43 [7\%] in the obeticholic acid $10 \mathrm{mg}$ group, and 42 [6\%] in the obeticholic acid $25 \mathrm{mg}$ group; serious adverse events: ten [2\%] placebo, nine [1\%] obeticholic acid $10 \mathrm{mg}$, and 13 [2\%] obeticholic acid $25 \mathrm{mg}$ ). Effects on glycaemic parameters were evaluated by baseline diabetes status (appendix $\mathrm{p}$ 14). In patients with type 2 diabetes, obeticholic acid treatment was associated with an early transient increase in glucose and $\mathrm{HbA}_{1 \mathrm{C}}$ with return to levels similar to placebo by month 6 . No clinically meaningful changes were noted in patients without diabetes. Blood pressure was generally stable, but variable, with no significant difference between treatment groups. Other vital signs were not affected by study treatments (data not shown).

Gallstone-related adverse events occurred in two $(<1 \%)$ patients in the placebo group, seven (1\%) in the obeticholic acid $10 \mathrm{mg}$ group, and 19 (3\%) in the obeticholic acid $25 \mathrm{mg}$ group. Pancreatitis, a more serious and potentially gallstone-related event, was rare and evenly distributed across treatment groups (one $[<1 \%]$ patient in each of the placebo and obeticholic acid $10 \mathrm{mg}$ groups and three $[<1 \%]$ patients in the obeticholic acid $25 \mathrm{mg}$ group). Hepatic serious adverse events were uncommon, and each case was reviewed by independent expert hepatologists. Although more events occurred in the obeticholic acid $25 \mathrm{mg}$ group (six [1\%] patients) than the obeticholic acid $10 \mathrm{mg}$ group (two $[<1 \%]$ patients) or placebo group (two $[<1 \%]$ patients), expert reviewers did not identify any consistent pattern of liver injury and all cases were associated with confounding concomitant medications or severe intercurrent illness.

Three deaths occurred on study: two in the placebo group (bone cancer and cardiac arrest) and one in the obeticholic acid $25 \mathrm{mg}$ group (glioblastoma). None were considered related to study treatment.

\section{Discussion}

To our knowledge, this study is the first positive phase 3 trial in NASH and represents a landmark in the development of new therapies for an increasingly common chronic liver disease. ${ }^{12-15}$ Treatment with obeticholic acid $25 \mathrm{mg}$ met the primary endpoint of improvement in fibrosis with no worsening of NASH in patients with stage F2 or F3 fibrosis, at the month-18 interim analysis. The robust antifibrotic effect of obeticholic acid was dose dependent and consistent across different patient populations and subgroups, and was further supported by fibrosis-related secondary endpoints, including an improvement in fibrosis of at least two stages. Per the draft guidance from the FDA on efficacy endpoints for clinical trials in NASH, improvement in fibrosis by at least one stage with no worsening of NASH is reasonably likely to predict clinical benefit. ${ }^{10}$ Patients with NASH have an almost 65 times greater risk of liver-specific mortality and almost three times greater risk of overall mortality compared with healthy individuals. ${ }^{14}$ Fibrosis has been shown to be the strongest histological predictor of liver-related adverse outcomes, including liver-related death. ${ }^{16-19}$ Treatment with obeticholic acid $25 \mathrm{mg}$ both improved fibrosis and prevented progression of fibrotic disease. To slow or reverse the progression of fibrosis is the ultimate goal of NASH treatment as fibrosis is the most reliable predictor of liver-related mortality and, once patients progress to cirrhosis, preventing complications of cirrhosis can become even more difficult. ${ }^{16,18}$

\begin{tabular}{|c|c|c|c|}
\hline & $\begin{array}{l}\text { Placebo } \\
(n=657)\end{array}$ & $\begin{array}{l}\text { Obeticholic acid } \\
10 \mathrm{mg}(n=653)\end{array}$ & $\begin{array}{l}\text { Obeticholic acid } \\
25 \mathrm{mg}(\mathrm{n}=658)\end{array}$ \\
\hline \multicolumn{4}{|l|}{ Treatment-emergent and serious adverse events } \\
\hline At least one treatment-emergent adverse event & $548(83 \%)$ & $579(89 \%)$ & $601(91 \%)$ \\
\hline \multicolumn{4}{|l|}{ Severity* } \\
\hline Mild & $160(24 \%)$ & $163(25 \%)$ & $130(20 \%)$ \\
\hline Moderate & $294(45 \%)$ & $323(49 \%)$ & $338(51 \%)$ \\
\hline Severe & $87(13 \%)$ & $89(14 \%)$ & $130(20 \%)$ \\
\hline Life-threatening & $5(1 \%)$ & $4(1 \%)$ & $2(<1 \%)$ \\
\hline Death & $2(<1 \%)$ & 0 & $1(<1 \%)$ \\
\hline Leading to treatment discontinuation & $41(6 \%)$ & $39(6 \%)$ & $83(13 \%)$ \\
\hline Serious adverse events & $75(11 \%)$ & $72(11 \%)$ & $93(14 \%)$ \\
\hline \multicolumn{4}{|c|}{ Adverse events occurring in $\geq 5 \%$ of patients in either obeticholic acid group } \\
\hline \multicolumn{4}{|l|}{ Skin and subcutaneous tissue disorders } \\
\hline Pruritus & $123(19 \%)$ & $183(28 \%)$ & $336(51 \%)$ \\
\hline Grade 1 (mild or localised) & $90(14 \%)$ & $113(17 \%)$ & $148(22 \%)$ \\
\hline Grade 2 (intense or wide spread) & $30(5 \%)$ & $67(10 \%)$ & $152(23 \%)$ \\
\hline $\begin{array}{l}\text { Grade } 3 \text { (intense or widespread and limit } \\
\text { activities of daily living) }\end{array}$ & $3(<1 \%)$ & $3(<1 \%)$ & $36(5 \%)$ \\
\hline \multicolumn{4}{|l|}{ Gastrointestinal disorders } \\
\hline Nausea & $77(12 \%)$ & $72(11 \%)$ & $83(13 \%)$ \\
\hline Constipation & $36(5 \%)$ & $65(10 \%)$ & $70(11 \%)$ \\
\hline Abdominal pain & $62(9 \%)$ & $66(10 \%)$ & $67(10 \%)$ \\
\hline Diarrhoea & $79(12 \%)$ & $44(7 \%)$ & $49(7 \%)$ \\
\hline Abdominal pain upper & $35(5 \%)$ & $46(7 \%)$ & $45(7 \%)$ \\
\hline Vomiting & $33(5 \%)$ & $34(5 \%)$ & $44(7 \%)$ \\
\hline Abdominal distension & $23(4 \%)$ & $31(5 \%)$ & $31(5 \%)$ \\
\hline \multicolumn{4}{|l|}{ Infections and infestations } \\
\hline Urinary tract infection & $49(7 \%)$ & $54(8 \%)$ & $62(9 \%)$ \\
\hline Upper respiratory tract infection & $44(7 \%)$ & $47(7 \%)$ & $54(8 \%)$ \\
\hline Nasopharyngitis & $41(6 \%)$ & $34(5 \%)$ & $45(7 \%)$ \\
\hline Bronchitis & $28(4 \%)$ & $34(5 \%)$ & $35(5 \%)$ \\
\hline Sinusitis & $35(5 \%)$ & $36(6 \%)$ & $30(5 \%)$ \\
\hline \multicolumn{4}{|l|}{ Investigations } \\
\hline LDL cholesterol increased & $47(7 \%)$ & $109(17 \%)$ & $115(17 \%)$ \\
\hline Blood cholesterol increased & $12(2 \%)$ & $30(5 \%)$ & $38(6 \%)$ \\
\hline \multicolumn{4}{|l|}{ Musculoskeletal and connective tissue disorders } \\
\hline Arthralgia & $55(8 \%)$ & $50(8 \%)$ & $50(8 \%)$ \\
\hline \multirow[t]{2}{*}{ Back pain } & $50(8 \%)$ & $56(9 \%)$ & $40(6 \%)$ \\
\hline & & \multicolumn{2}{|c|}{ (Table 3 continues on next page) } \\
\hline
\end{tabular}




\begin{tabular}{|c|c|c|c|}
\hline & $\begin{array}{l}\text { Placebo } \\
(n=657)\end{array}$ & $\begin{array}{l}\text { Obeticholic acid } \\
10 \mathrm{mg}(n=653)\end{array}$ & $\begin{array}{l}\text { Obeticholic acid } \\
25 \mathrm{mg}(\mathrm{n}=658)\end{array}$ \\
\hline \multicolumn{4}{|c|}{ (Continued from previous page) } \\
\hline \multicolumn{4}{|c|}{ Metabolism and nutrition disorders } \\
\hline Hyperlipidaemia & $18(3 \%)$ & $42(6 \%)$ & $55(8 \%)$ \\
\hline Diabetes & $36(5 \%)$ & $46(7 \%)$ & $45(7 \%)$ \\
\hline Hypercholesterolaemia & $14(2 \%)$ & $35(5 \%)$ & $29(4 \%)$ \\
\hline \multicolumn{4}{|c|}{ General disorders and administration site conditions } \\
\hline Fatigue & $88(13 \%)$ & $78(12 \%)$ & $71(11 \%)$ \\
\hline \multicolumn{4}{|l|}{ Nervous system disorders } \\
\hline Headache & $51(8 \%)$ & $42(6 \%)$ & $34(5 \%)$ \\
\hline Dizziness & $28(4 \%)$ & $32(5 \%)$ & $25(4 \%)$ \\
\hline \multicolumn{4}{|c|}{ Respiratory, thoracic, and mediastinal disorders } \\
\hline Cough & $27(4 \%)$ & $29(4 \%)$ & $38(6 \%)$ \\
\hline \multicolumn{4}{|l|}{ Vascular disorders } \\
\hline Hypertension & $28(4 \%)$ & $36(6 \%)$ & $39(6 \%)$ \\
\hline $\begin{array}{l}\text { Table is arranged by descendi } \\
\text { in the obeticholic acid } 25 \mathrm{mg} \\
\text { *Patients reporting more tha }\end{array}$ & $\begin{array}{l}\text { organ class } \\
\text { ng order of in } \\
\text { ted only onc }\end{array}$ & $\begin{array}{l}\text { referred term within } \\
\text { ce in the obeticholic } \\
\mathrm{g} \text { the highest severit }\end{array}$ & $\begin{array}{l}\text { system organ class) } \\
\text { acid } 10 \mathrm{mg} \text { group. } \\
\text { y. }\end{array}$ \\
\hline
\end{tabular}

Although the percentage of patients achieving NASH resolution was not significant between obeticholic acid and placebo, more patients receiving obeticholic acid $25 \mathrm{mg}$ showed improvements in hepatocellular ballooning and lobular inflammation, the two key histological features of the prespecified NASH resolution endpoint. These data are relevant given that features of steatohepatitis, such as hepatocellular ballooning, are predictive of increased liver-related events and reduced liver transplant-free survival. ${ }^{19}$ Additionally, more patients receiving obeticholic acid $25 \mathrm{mg}$ had an improvement of at least two points in NAS with no worsening of fibrosis, the primary endpoint traditionally used in phase 2 studies such as FLINT ${ }^{7}$ and PIVENS ${ }^{20}$ indicating that obeticholic acid reduces NASH disease activity.

Twice as many obeticholic acid $25 \mathrm{mg}$ patients compared with placebo achieved NASH resolution as determined by the post-hoc pathologist diagnostic assessment of the absence of definite steatohepatitis at month 18 . This evaluation was based on an assessment of the overall pattern of histological lesions or injury, as opposed to the more rigid categorical scoring system of the prespecified methodology described above. This finding has clinical relevance given that this definition is commonly used to diagnose NASH in clinical practice, as well as in natural history studies evaluating the correlation of definite NASH and mortality. ${ }^{16}$ The assessment of NASH resolution based on NAS parameters appears to be more rigid and might be associated with greater intra-rater and interrater variability compared with the diagnostic classification of NASH. ${ }^{21}$ The NAS, a tool designed to measure disease activity and severity in NASH, is distinct from a clinical diagnosis of definite steatohepatitis. In an investigation into the relationship between NAS and the diagnosis of steatohepatitis, threshold values of NAS did not always correlate with pathologist overall assessment of presence of NASH. ${ }^{22}$ Therefore, as the field continues to evolve, it might be more appropriate to establish the presence or absence of NASH using histological diagnostic criteria as an endpoint, as has been done by the National Institute of Diabetes and Digestive and Kidney Diseases' NASH Clinical Research Network in the past.

In addition to consistent improvements in multiple histological parameters, improvement in liver health was also evident based on clinically meaningful, dosedependent improvements in markers of liver injury (ALT and AST) and oxidative stress (GGT). The modest increases in ALP are consistent with earlier observations and are associated with an on-target effect of farnesoid $\mathrm{X}$ receptor activation.

Lifestyle modifications including weight loss have been shown to be an effective non-pharmacological therapy for NAFLD. Weight loss greater than $7 \%$ has been associated with improvement in NAS and weight loss of at least $10 \%$ with improvement in fibrosis. ${ }^{23}$ Obeticholic acid-treated patients experienced weight loss of approximately $2 \%$, an amount lower than that expected to have an effect on histological parameters of NASH. Although modest, the effect of obeticholic acid on weight is important to note given the prevalence of obesity and metabolic abnormalities in this population.

Based on a substantial safety population including almost 2000 patients, of whom approximately 900 were exposed for at least 18 months, obeticholic acid was generally well tolerated. Most adverse events were mild to moderate in severity and were generally consistent with the known safety profile of obeticholic acid.? As previously seen, mild-to-moderate pruritus was the most commonly reported adverse event, with a dose-dependent incidence. More patients in the obeticholic acid $25 \mathrm{mg}$ group experienced pruritus that led to treatment discontinuation; however, most randomised patients were ongoing in the study through at least month 18 and the overall treatment discontinuation rate was similar to placebo. The impact of pruritus in this study on patient-reported outcomes and its relationship to obeticholic acid is being investigated. ${ }^{24}$ The incidence of hepatic adverse events was balanced across treatment groups, and serious hepatic events were rare; although numerically more occurred in the obeticholic acid $25 \mathrm{mg}$ treated group, there was no clear pathological pattern seen consistently among these serious adverse events and all cases were confounded by concomitant medications or severe intercurrent illness. Treatment with obeticholic acid was associated with serum lipid changes that were consistent with a class effect of farnesoid $\mathrm{X}$ receptor activation, as well as small and generally transient increases in glycaemic parameters. Such increases were manageable by clinical practice measures. The effect of lipid changes on cardiovascular risk should be assessed in the context of other obeticholic 
acid-related reductions in risk factors, including a decrease in bodyweight, serum triglyceride levels, and GGT, a promising marker for assessing cardiovascular risk, as well as improvements in liver fibrosis, which might have a downstream effect on cardiovascular risk. ${ }^{19,25-27}$ The incidence of cardiovascular adverse events and serious adverse events was low and similar across treatment groups and continues to be monitored in the outcomes portion of the study.

The results of the interim analysis reported here are clinically relevant in the context of fibrosis due to NASH but might underestimate the long-term benefit of obeticholic acid on the target illness. Improvement in fibrosis, a generally slow process, was observed at the month-18 interim analysis of the ongoing study, and the effect size might increase with prolonged therapy. This has been shown with other interventions that reported improvement in fibrosis at early timepoints with a greater effect over the longer term. For example, tenofovir treatment resulted in 10\% fewer patients with hepatitis B virus-associated advanced fibrosis or cirrhosis after the first year of treatment ( $28 \%$ vs $38 \%$ at baseline). ${ }^{28}$ In the tenofovir study, patients continued to improve on treatment, and the proportion of patients with advanced fibrosis or cirrhosis declined to $12 \%$ at year $5 .{ }^{27}$ In REGENERATE, the continuing improvement in liver enzyme markers of fibrosis such as ALT and AST suggest the potential for further increase in antifibrotic response. Data from the ongoing long-term outcomes portion of the study will inform whether prolonged therapy will result in a greater antifibrotic benefit.

This is a prespecified interim analysis of an ongoing study and the histological outcomes, in particular fibrosis stage, have been shown to be reasonably likely to predict clinical outcomes and therefore supports regulatory submission based upon the conditional approval pathway. However, a limitation of this analysis is that clinical outcomes data are not yet available. Additionally, the study population consists of patients selected on the basis of biopsy evidence of NASH and fibrosis; however, physicians increasingly rely on non-invasive means to diagnose and stage patients with NASH with fibrosis, which might have an implication for the real world relevance of the results. Non-invasive assessments of liver fibrosis such as the Fibrosis-4 Index and transient elastography are potentially more sensitive continuous parameters than categorical assessment of change in histological fibrosis stage; these were assessed throughout the study and will be reported at a later date. Finally, this interim analysis was completed with evolving regulatory authority guidances in which, for example, the definition of histological NASH resolution has changed, with the implication that future pivotal study designs could continue to be modified.

In conclusion, the totality of data from the month-18 interim analysis of this phase 3 study provides strong evidence that obeticholic acid treatment improves clinically significant histological endpoints deemed reasonably likely to predict clinical benefit, and affirms the positive benefit-risk ratio of obeticholic acid for the treatment of NASH with fibrosis. Beneficial effects of obeticholic acid on fibrosis and key components of NASH disease activity were robust, based on the observed consistency of results across multiple histological endpoints with reproducible response ratios, as well as the evident dose-response and markedly consistent benefit across analysis populations. Treatment with obeticholic acid had a beneficial effect on other markers of hepatocellular injury (ALT and AST) and oxidative stress (GGT). Obeticholic acid was generally well tolerated, with a profile that is generally consistent with prior studies. Following the month-18 interim analysis, this study continues in a blinded fashion, and patients will be followed up over an extended period through clinical outcomes (including all-cause mortality and liver-related clinical outcomes) and long-term safety, to confirm clinical benefit. In a chronic liver disease with no approved therapies and potential for serious sequelae, these findings provide compelling evidence that patients with non-cirrhotic advanced fibrosis due to NASH might benefit from obeticholic acid treatment.

Contributors

VR, AJS, and ZMY participated in initial study design in collaboration with the sponsor (DSha, LM, RS). AJS, MR, PB, QMA, RL, SH, VR, ZG, and ZMY (chair) make up the steering committee, which is responsible for ongoing conduct of the study. ZMY, VR, RL, MR, QMA, AG, SB, PNN, DShe, JT, WK, EL, MFA, KVK, MYS, AJM-L, JB, PM, EB, GM, AO, HC-P, IG, DO, LLG, and J-FD participated in data collection. AJS, MR, PB, QMA, PNN, RL, SH, VR, MFA, DSha, JC, LZ, LM, RS, ZG, and ZMY participated in data analysis and interpretation. All authors participated in manuscript development.

\section{Declaration of interests}

All declarations are outside of the submitted work. ZMY has received research funds or consultation fees from Gilead Sciences, NovoNordisk, Intercept, Novartis, Terns, Viking, Siemens, and Echosens. QMA is coordinator of the EU IMI2-funded LITMUS consortium. His institution has received research grants from AbbVie, Allergan/Tobira, AstraZeneca, GlaxoSmithKline, Glympse Bio, Novartis Pharma, Pfizer, and Vertex. He has performed consultancy on behalf of Newcastle University for Abbott Laboratories, Acuitas Medical, Allergan/Tobira, Blade, BNN Cardio, Cirius, CymaBay, EcoR1, E3Bio, Eli Lilly \& Company, Galmed, Genfit, Gilead, Grunthal, HistoIndex, Indalo, Imperial Innovations, Intercept Pharma Europe, Inventiva, IQVIA, Janssen, Kenes, Madrigal, MedImmune, Metacrine, NewGene, NGMBio, North Sea Therapeutics, Novartis, Novo Nordisk, Pfizer, Poxel, ProSciento, Raptor Pharma, Servier, and Viking Therapeutics. He has received speaker fees from Abbott Laboratories, Allergan/Tobira, Bristol-Myers Squibb, Clinical Care Options, Falk, Fishawack, Genfit, Gilead, Integritas Communications, and MedScape, and royalties from Elsevier. AJS is president of Sanyal Bio. He has stock options in Indalo, Durect, Tiziana, Exhalenz, and Northsea. He is a consultant to Gilead, Allergan, Bristol-Myers Squibb, Pfizer, Merck, Galmed, Novartis, Novo Nordisk, Lilly, Siemens, Genentech, Boehringer Ingelhiem, Glympse Bio, Genfit, Coherus, Surrozen, Poxel, 89 Bio, Perspectum, AstraZeneca, Medimmune, and Lipocine. He is an unpaid consultant to Intercept, Zydus, Echosense, Immuron, Madrigal, Galectin, Blade, Pliant, Albireo, and AMRA. RL serves as a consultant or advisory board member for Arrowhead Pharmaceuticals, AstraZeneca, Bird Rock Bio, Boehringer Ingelheim, Bristol-Myers Squibb, Celgene, Cirius, CohBar, Conatus, Eli Lilly, Galmed, Gemphire, Gilead, Glympse bio, GNI, GRI Bio, Intercept, Ionis, Janssen, Merck, Metacrine, NGM Biopharmaceuticals, Novartis, 
Novo Nordisk, Pfizer, Prometheus, Sanofi, Siemens, and Viking Therapeutics. His institution has received grant support from Allergan, Boehringer-Ingelheim, Bristol-Myers Squibb, Cirius, Eli Lilly and Company, Galectin Therapeutics, Galmed Pharmaceuticals, GE, Genfit, Gilead, Intercept, Grail, Janssen, Madrigal Pharmaceuticals, Merck, NGM Biopharmaceuticals, NuSirt, Pfizer, pH Pharma, Prometheus, and Siemens; he is also co-founder of Liponexus. PNN reports consultancy or speaker fees on behalf of the University of Birmingham from Boehringer Ingelheim, Gilead, Pfizer, Affimmune, Intercept, Johnson \& Johnson, Novo Nordisk, Shire, and Poxel Pharmaceuticals. His institution receives grant funding from Pharmaxis, Boehringer Ingelheim, and Novo Nordisk. All remaining authors declare no competing interests.

\section{Data sharing}

The authors declare that all data supporting the findings of this interim analysis are available within the Article and its appendix. The study is ongoing at the time of publication and blinded at the individual level; patient-level data therefore will not be available until the end-of-study analysis.

\section{Acknowledgments}

Financial support for medical editorial assistance was provided by Intercept Pharmaceuticals. We thank medical writers William Sinkins (ProEd Communications) and Kjersti Swearingen (Intercept Pharmaceuticals) for editorial assistance with this manuscript. PNN was supported by the National Institute of Health Research (NIHR) Birmingham Biomedical Research Centre. The views expressed are those of the authors and not necessarily those of the National Health Service, the NIHR, or the UK Department of Health.

\section{References}

1 Estes C, Razavi H, Loomba R, Younossi Z, Sanyal AJ. Modeling the epidemic of nonalcoholic fatty liver disease demonstrates an exponential increase in burden of disease. Hepatology 2018; 67: 123-33.

2 Modica S, Gadaleta RM, Moschetta A. Deciphering the nuclear bile acid receptor FXR paradigm. Nucl Recept Signal 2010; 8: e005.

3 Fiorucci S, Antonelli E, Rizzo G, et al. The nuclear receptor SHP mediates inhibition of hepatic stellate cells by FXR and protects against liver fibrosis. Gastroenterology 2004; 127: 1497-512.

4 Gadaleta RM, van Erpecum KJ, Oldenburg B, et al. Farnesoid X receptor activation inhibits inflammation and preserves the intestina barrier in inflammatory bowel disease. Gut 2011; 60: 463-72.

5 Wang YD, Chen WD, Wang M, Yu D, Forman BM, Huang W. Farnesoid $\mathrm{X}$ receptor antagonizes NF- $\mathrm{BB}$ in hepatic inflammatory response. Hepatology 2008; 48: 1632-43.

6 Mudaliar S, Henry RR, Sanyal AJ, et al. Efficacy and safety of the farnesoid $\mathrm{X}$ receptor agonist obeticholic acid in patients with type 2 diabetes and nonalcoholic fatty liver disease. Gastroenterology 2013; 145: 574-82.

7 Neuschwander-Tetri BA, Loomba R, Sanyal AJ, et al. Farnesoid X nuclear receptor ligand obeticholic acid for non-cirrhotic, non-alcoholic steatohepatitis (FLINT): a multicentre, randomised, placebo-controlled trial. Lancet 2015; 385: 956-65.

8 Nevens F, Andreone P, Mazzella G, et al. A placebo-controlled trial of obeticholic acid in primary biliary cholangitis. N Engl J Med 2016 375: 631-43.

9 European Medicines Agency. Reflection paper on regulatory requirements for the development of medicinal products for chronic non-infectious liver diseases (PBC, PSC, NASH). Nov 19, 2018. https://www.ema.europa.eu/en/draft-reflection-paperregulatory-requirements-development-medicinal-products-chronicnon-infectious (accessed Jan 28, 2019).

10 US Department of Health and Human Services. Noncirrhotic nonalcoholic steatohepatitis with liver fibrosis: developing drugs for treatment. Guidance for industry. December, 2018. https://www.fda gov/regulatory-information/search-fda-guidance-documents/ noncirrhotic-nonalcoholic-steatohepatitis-liver-fibrosis-developingdrugs-treatment (accessed Jan 28, 2019).
11 Ratziu V, Sanyal AJ, Loomba R, et al. REGENERATE: Design of a pivotal, randomised, phase 3 study evaluating the safety and efficacy of obeticholic acid in patients with fibrosis due to nonalcoholic steatohepatitis. Contemp Clin Trials 2019; 84: 105803.

12 Younossi Z, Tacke F, Arrese M, et al. Global perspectives on nonalcoholic fatty liver disease and nonalcoholic steatohepatitis Hepatology 2019; 69: 2672-82.

13 Younossi ZM. Non-alcoholic fatty liver disease-a global public health perspective. J Hepatol 2019; 70: 531-44.

14 Younossi ZM, Koenig AB, Abdelatif D, Fazel Y, Henry L, Wymer M. Global epidemiology of nonalcoholic fatty liver disease-meta-analytic assessment of prevalence, incidence, and outcomes. Hepatology 2016; 64: 73-84.

15 Younossi ZM, Stepanova M, Younossi Y, et al. Epidemiology of chronic liver diseases in the USA in the past three decades. Gu 2019; published online July 31. DOI:101.136/gutjnl-20193-18813.

16 Dulai PS, Singh S, Patel J, et al. Increased risk of mortality by fibrosis stage in nonalcoholic fatty liver disease: systematic review and meta-analysis. Hepatology 2017; 65: 1557-65.

17 Hagstrom H, Nasr P, Ekstedt M, et al. Fibrosis stage but not NASH predicts mortality and time to development of severe liver disease in biopsy-proven NAFLD. J Hepatol 2017; 67: 1265-73.

18 Younossi ZM, Stepanova M, Rafiq N, et al. Pathologic criteria for nonalcoholic steatohepatitis: Interprotocol agreement and ability to predict liver-related mortality. Hepatology 2011; 53: 1874-82.

19 Angulo P, Kleiner DE, Dam-Larsen S, et al. Liver fibrosis, but no other histologic features, is associated with long-term outcomes of patients with nonalcoholic fatty liver disease. Gastroenterology 2015; 149: 389-97.

20 Sanyal AJ, Chalasani N, Kowdley KV, et al. Pioglitazone, vitamin E, or placebo for nonalcoholic steatohepatitis. N Engl J Med 2010; 362: $1675-85$.

21 Kleiner D, Brunt E, Van Natta M, et al. Design and validation of a histological scoring system for nonalcoholic fatty liver disease. Hepatology 2005; 41: 1313-21.

22 Brunt EM, Kleiner DE, Wilson LA, Belt P, Neuschwander-Tetri BA Network NCR. Nonalcoholic fatty liver disease (NAFLD) activity score and the histopathologic diagnosis in NAFLD: distinct clinicopathologic meanings. Hepatology 2011; 53: 810-20.

23 Hannah WN Jr, Harrison SA. Effect of weight loss, diet, exercise, and bariatric surgery on nonalcoholic fatty liver disease. Clin Liver Dis 2016; 20: 339-50.

24 Younossi ZM, Stepanova M, Younossi I, Racila A. Validation of chronic liver disease questionnaire for nonalcoholic steatohepatitis in patients with biopsy-proven nonalcoholic steatohepatitis. Clin Gastroenterol Hepatol 2019; 17: 2093-100.e3.

25 Lee DS, Evans JC, Robins SJ, et al. Gamma glutamyl transferase and metabolic syndrome, cardiovascular disease, and mortality risk: the Framingham Heart Study. Arterioscler Thromb Vasc Biol 2007; 27: $127-33$.

26 Wannamethee G, Ebrahim S, Shaper AG. Gamma glutamyltransferase: determinants and association with mortality from ischemic heart disease and all causes. Am J Epidemiol 1995; 142: 699-708.

27 Budoff M. Triglycerides and triglyceride-rich lipoproteins in the causal pathway of cardiovascular disease. Am J Cardiol 2016; 118: $138-45$

28 Marcellin P, Gane E, Buti M, et al. Regression of cirrhosis during treatment with tenofovir disoproxil fumarate for chronic hepatitis $B$ : a 5-year open-label follow-up study. Lancet 2013; 381: 468-75. 